\title{
Ética y política: Entre la utopía y la justicia social
}

- Antônio Sidekum

Doctorado por la Universidad de Bremen, Alemana; POST-Doctorado por la Universidad de Leprig, Alemania y por The Cathoulc Universtiy of America, Washington, Ee.UU.

PROFESOR DE FILOSOFIA DE LA USINOS.

- Traducción de Luis Alvarengo

\section{Cuestiones preliminares}

Se pretende introducir el tema Ética y política: entre la utopia y la justicia social, partiendo del análisis de dos perspectivas críticas que, aún ahora, parecen ser las más significativas para reflexionar sobre las innumerables situaciones conflictivas en el campo de la política y la justicia social en la actualidad. La primera hace referencia al pensamiento de Walter Benjamin', que trata del materialismo histórico, como pieza propulsora del desenvolvimiento del concepto de política y de progreso. La segunda perspectiva parte del pensamiento sociopolítico de Immanuel Wallerstein ${ }^{2}$, cuando cuestiona las altemativas todavía posibles en tanto que posibilidades para una política contemporánea, es decir, de un sistema mundial globalizado, y que esté ligada con los derechos humanos y el bien, así como con un mundo más justo para las sociedades.

Walter Benjamin, al introducir su texto crítico sobre el concepto de historia, que busca apuntar para una comprensión de la dimensión de la ética y la política nos presenta, entre otras cosas, el siguiente pasaje: "Conocemos la historia de un autómata construido de tal suerte que podia responder a cada lance de un jugador de ajedrez con un contralance que le aseguraba la victoria. Un fantoche vestido a la usanza turca, con un narguile en los labios, se sentaba delante del tablero, 
colocado encima de una enorme mesa. Un sistema de espejos creaba la ilusión de que la mesa era totalmente visible en todos sus detalles. En realidad, un enano deforme se escondía en ella: un maestro de ajedrez, que dirigía con los cordeles la mano del fantoche. Podemos imaginar una contrapartida filosófica de ese mecanismo. El fantoche llamado "materialismo histórico" ganará siempre. Él puede enfrentar cualquier desafio, siempre y cuando ponga la teología a su servicio. Hoy, ella es reconocida públicamente como pequeña y fea y no se atreve a dar la cara".'.

En la perspectiva de este análisis de la dimensión ética y política de Walter Benjamin, debemos subrayar importantes aspectos que intervienen en la realización del mundo político volcado hacia la ética, como una posibilidad inherente al proceso de desenvolvimiento de la vida social, que posee, como desiderata última y original la fundamentación teológica, en el sentido de la concepción axiológica de Adam Smith de la "mano invisible". Según Smith, podríamos resumir, todo individuo trabaja necesariamente en el sentido de hacer que el rendimiento anual de la sociedad sea lo mayor posible. En verdad, este individuo generalmente no tiene intenciones de promover el interés público y tampoco sabe en qué medida lo promueve. Al preferir el sostenimiento de la actividad doméstica, que es exterior, él tiene en vista tan sólo su seguridad. Y, al dirigir esa actividad de tal manera que su producción alcance el mayor valor posible, él tiene en vista únicamente su propio lucro y, en este caso, como en muchos otros, está guiado por una mano invisible para promover un fin que no formaba parte de sus intenciones. $\mathrm{Y}$ el hecho de que este fin no forme parte de sus intenciones no siempre es lo peor para la sociedad. Al buscar sus propios intereses, frecuentemente, el individuo promueve el interés de la sociedad de manera más eficiente que si tuviera realmente la intención de promoverlo. Así, debería instaurarse un principio contradictorio que moviese todas las energías del humano para trascender la nuda y pura naturaleza de los intereses individuales para alcanzar la realización de la justicia social. Esa dimensión se vuelve una comprensión escatológica salvacionista de la historia. Muchas veces, Benjamin se vale de ese lenguaje casi apoteósico de la fuerza del materialismo histórico sobre la formación política del ser humano. Se trata de una referencia inenarrable de la conciencia de la tensión histórica entre el Paraíso perdido y la esperanza de poder recuperarlo algún día. Existe en la lectura de ese pasaje una presencia de la fuerza que se conjuga como contrapunto del proceso de la historia que es el materialismo histórico y su respectivo sustentáculo que acontece en la dimensión teológica. La teología, entre tanto, es fundamental para la comprensión del vacío sufrido por la política. "Conviene mantener la distinción de diferentes niveles en los cuales se mueve el «discurso teológico». Un nivel es el de la simple constatación de la metamorfosis de los dioses, es decir, de la variación de las imágenes con respecto a las divinidades, que penetran en teorías y políticas económicas. En ese nivel, además de lo teológico, cualquier científico social puede perfectamente manifestar su interés en el asunto. Cuando se apunta 
hacia conflictos y antagonismos entre los dioses, ya nos encontramos en otro nivel: el de la lucha de los dioses. Siempre hubo dioses conflictivos entre sí, porque son conflictivas entre ellas las concepciones de que los seres humanos, apoyados en sus imaginaciones teológicas, tienen al respecto de su vida en la historia... El tercer nivel es, pues, el del decubrimiento de las utilidades específicas de los dioses. Son útiles para determinadas funciones... Y en el cuarto nivel aparece el discurso teológico sobre la idolatría, esto es, sobre los ídolos que matan, porque exigen sacrificios"."

En la atribución del materialismo histórico, tratamos de las posibilidades reales de la política en cuanto entidad que realiza la satisfacción de las necesidades ya comprendidas como supraculturales en la realidad histórica del ser humano: Encontrar las condiciones de ideación y de manipulación de la naturaleza que contiene el mundo ambiente del ser humano. Así, el proceso cultural, como fenómeno de hominización ${ }^{6}$ tiene como principio fundante la ideación y la transformación irreversible del mundo humano. Se trata de la satisfacción de las primeras necesidades y de la satisfacción de las necesidades trascendentes de la vida humana, de las necesidades más intrigantes de las relaciones interhumanas y del desenvolvimiento de la propia historia. Según Marx, "un valor de uso o un bien sólo posee valor, porque en él está corporizado, materializado, el trabajo humano abstracto. Toda la fuerza de trabajo de la sociedad —que se revela en los valores del mundo, de las mercancías - vale, aquí, tan sólo por la fuerza de trabajo, aunque se constituya de innumerables fuerzas de trabajo individuales. Cada una de esas fuerzas individuales de trabajo se equipara a las demás, en la medida en que posea el carácter de una fuerza promedio de trabajo social y actúe como esa fuerza promedio, necesitando, en consecuencia, apenas del tiempo de trabajo promedio necesario o socialmente necesario para la producción de una mercancía". En la perspectiva benjaminiana, leemos, por tanto, la concepción marxista sobre la conciencia histórica, que se instaura como conciencia humana última que es la transformación del mundo y de su propia historia, como podemos inferir de las tesis fundamentales de Karl Marx ${ }^{k}$. Esa conciencia histórica implica una acción que el lenguaje traduce, como objeto transformador en la manipulación del mundo humano, sin ser meramente una reducción eidética fenomenológica. Pero el lenguaje se entiende como condición primera de la afirmación de la historicidad humana, tal como podemos inferir en Ludwig Feuerbach". El lenguaje como esencia distintiva del ser humano, como acto de trabajo" en la realización de sus actividades culturales, necesariamente implica, como en el pensamiento de Aristóteles", la condición para la vida política del ser humano.

Del pasaje de Walter Benjamin para el pensamiento político contemporáneo, nos quedamos con innumerables variables y condicionamienntos para una mejor comprensión del paradigma político como promotor de garantías de realización de la justicia social. En esa perspectiva, debemos considerar las enormes y rápidas transformaciones históricas subyacentes al siglo XX, principalmente, en las 
dos últimas décadas: Por un lado, analizamos la derrota del socialismo real y la crisis del capitalismo contemporáneo, esto en el campo político, y por otro lado, tratamos del extraordinario desarrollo de la biotecnocultura, cuya repercusión es un extraordinario desafío para la ética. Aquí vamos a ponderar el trabajo de Immanucl Wallerstein, que se refiere al período que ćl mismo denomina "posliberalismo"'?2. tomando en cuenta la agonía del neoliberalismo. El autor valora sobre esa fase histórica el que el liberalismo presentaba determinadas consignaciones en períodos pasados, tales como la consolidación de los llamados ideales de democracia y de la consideración de los derechos humanos reclamados y proclamados en algunos movimientos revolucionarios de los siglos XVIII y XIX, y acentuando un largo período de sueños utópicos que traían en su arcano la realización de la plenitud de la justicia humana, como conquista de los derechos humanos. Ese período, según Wallerstein, es de enormes frustraciones y de desesperanza una vez que, concomitante a la utopía de la ćpoca, se desarrolla la consolidación de los ideales liberales bajo una nueva perspectiva política, teniendo como enlace social la nucva lorma e ideología cconómicas, sea cual sea el poder económico capitalista que instaura en sí mismo la lundamentación de la ética, sin considerar más los ideales históricos que orientaban la concepción de democracia, vaciando con ese poder la mera posibilidad del Estado como gerente de la justicia social. El autor se relïere a los cincuenta años venideros como un período de las más trágicas experiencias de carniccría de los principios democráticos y de las fonnas inescrupulosas de las luerzas cconómicas - la lorma del capitalismo-, en relación con la destrucción del medio ambiente y de la lorma que tomará la violencia institucionalizada en lo que se refiere a las poblaciones excluidas en todos los rincones del planeta ${ }^{13}$.

Las últimas décadas del siglo $\mathrm{XX}$ confirmaron que vivimos una era de extre$\operatorname{mos}^{14}$. Esos extremos sumergen al ser humano de manera desesperada e indefensa en una era de certezas lúgubres en lo que respecta a la defensa de la democracia y los derechos humanos. Asistimos a la inmersión de la humanidad toda en totalitarismos extremados y lundamentalismos sanguinarios. Observamos que la Humanidad nunca lue coctánea de tanto progreso científico y tecnológico como en ese siglo. Somos contemporáneos de la mayoría absoluta de los científicos de toda la historia. El siglo XX experimentó la más profunda tragedia de la concepción de progreso ${ }^{14}$. La tecnología alcanzó dominios sobre la naturaleza que antes parecían utopías y algo jamás imaginable por el ser humano. El poder que el ser humano como rasgo de su personalidad, se vuelve un poder que huye de los límites del equilibrio y de la armonía de la vida humana para con la naturaleza de la cual lorma parte. En nombre del progreso, la naturaleza pasa a ser violentada, expoliada y masacrada, volviéndose, de esta manera, un mundo hostil para cl propio ser humano. Esa misma concepción del poder violento de la biotecnocultura se extiende sobre las más diversas formas de las ideologías totalitarias que chocan con las concepciones políticas en todo el mundo, defendiendo la verdad absoluta aun cuando esto implique aniquilar a multitudes de seres humanos

\section{2}

Realidad 82, 2001 
bajo el hierro incandescente de la destrucción, por la muerte y por la negación absoluta e irrestricta de los derechos humanos más fundamentales. La política pierde, de este modo, su carácter utópico y escatológico, en el sentido de la República de Platón, así como también pierde su sentido armónico y plástico, que procede de la concepción de Aristóteles de armonía de las múltiples relaciones humanas originadas y fundamentadas en la concepción ideal y real de la Polis, que a través del Estado desarrolla la justicia social para los ciudadanos. El proceso de transformación tecnológica y científica sostenido por el poder económico, que a su vez se traduce en forma de violencia institucionalizada y por el poder teológico de la mano invisible que mueve el mercado, según Adam Smith, y cuya idolatría del mercado exige sacrificios de sangre humana. Horkheimer y Adorno nos heredaron una breve reflexión sobre el sacrificio como lógica implícita en las relaciones mercantiles del capitalismo. el intercambio mercantil puede ser analizado, según ellos, como una secularización del sacrificio. El fetichismo ${ }^{16}$ de las relaciones mercantiles está compuesto de apariencias y abstracciones del intercambio, oculto por un manto reconciliador. De esta manera, las abstracciones del intercambio encarnan, en sus apariencias, la misma relación natural $\multimap$ de "naturalización de la historia"- que el sacrificio cumple de acuerdo con sus visiones religiosas. Lo que es contradictorio se vuelve a armonizar, tanto por el rito sacrificial como por el ritual de intercambio ${ }^{17}$.

\section{Los desafios éticos en la era de la globalización}

Pasamos, ahora, de las reflexiones críticas de Walter Benjamin a enunciar algunas implicaciones breves del pensamiento político de Immanuel Wallerstein accrca del papel de la política, según su expresión consagrada, en la era posliberal. En cste sentido, debemos tomar en cuenta las grandes transformaciones que la humanidad sufrió en las últimas décadas del siglo XX, principalmente en el campo político. El pensamiento político se desarrolla en los senderos de la liberlad económica como expresión imprescindible para la democracia y, finalmente, se introduce un nuevo acento en el pensamiento político, como expresión de la democracia, que es la identidad, es decir, se lucha vigorosamente en todos los segmentos de la sociedad política por el derecho de tener una identidad sustentada históricamente. Esta perspectiva histórica se puede recorrer por los grandes bloques temáticos, que caracterizan las últimas décadas, tal como ocurre en el campo sociopolítico, el de las revoluciones, algunas de ellas soñadas utópicamente y otras, llevadas a cabo con un despliegue de violencia inenarrable e imposible de conmensurar, v. gr.: la revolución de Mao Tse Tung, del Kmer Rojo de Laos y la rebeldía de los jóvenes en 1968, protestando contra la Guerra de Vietnam y exigiendo nuevos paradigmas de estilo de vida, tales como los movimientos de contracultura y la dimensión socioeconómica, como sería el caso de la teoría de la dependencia, como marco referencial para el análisis de la siluación internacional. histórica y económica, en aquel momento.

Ética y política: Entre la utopia y la justicia social 
La siguiente crisis fue la crisis política referida al propio modo de ser y de conducir un modelo político y económico, fundamentalmente, la recuperación de la economía europea y la implantación de la modernización en países subdesarrollados y en los países llamados en vias de desarrollo, a través de dictaduras militares sostenidas por la burguesía corrupta. Y, finalmente, en el último conflicto de las ideologías, precedidas por la Primavera de Praga, la Glasnost y la Perestroika, que culminan con el fin de la guerra fría, cuando emerge el debate en torno de la identidad. El problema de la identidad, como forma de repensar el modelo político, surge siempre, en muchos contextos de enormes ambigüedades, sea bajo la forma de multiculturalismo, de interculturalidad o también de transculturación.

Por globalización entendemos los procesos de cambios en la vida contemporánea de la economía, la política y de la red cibernética de comunicación social. Debemos, además, acentuar que el concepto de globalización no se refiere tan sólo a los aspectos de la economía mundial, sino que trae con vehemencia, en su envergadura, nuevas perspectivas, que son referentes, principalmente, de los riesgos que asumimos en común de cara a las constantes catástrofes a las que podemos ver gracias a los medios de comunicación social, catástrofes que se suscitan en el sistema de redes de transporte, amenazas concomitantes a los experimentos científicos y tecnológicos. Este concepto refleja, fundamentalmente, una novedad constante en la vida humana. El mismo nivel de inquietud se da mediante la angustia existencial, derivada de la consciencia inmoral ${ }^{18}$ y del sentido de una inmensa capacidad abarcadora de la aplicación de las redes de comunicación mundial, así como de los horizontes del mercado económico-financiero mundial, que a cada instante podrá representar una verdadera tragedia para los países que tienen a sus economías presas en el sistema mundial, ambos vistos de manera más tangible e inmediata, en un presente sin pasado, es decir, en un presente sin profundas raíces en el pasado, de lo que sucedió en el período de expansión y de conquistas europeas y, respectivamente, en los primeros estadios de la Modernidad. Enrique Dussel enfoca la Modernidad, en su historización de la ética, bajo tres límites: La muerte de la vida en su totalidad, a través del uso indiscriminado del poder de tecnologías antiecológicas, constituidas progresivamente bajo el único criterio de la gerencia adıninistrativa y cuantitativa del sistema mundial en la Modernidad. El segundo límite de ésta trata de un proceso de destrucción de la humanidad en sí misma, a través del proceso violento y descontrolado de la revolución industrial. El tercer límite de la Modernidad es la subsunción de las poblaciones, economías, naciones y culturas. Dussel lo manifiesta así1": "The first absolute limit: the death of life in its totality, through the indiscriminate use of anti-ecological technology constituted progressively through the sole criterion of the quantitative management of the world system in the modernity: the increase in the rate of profit. But capital cannot limit itself. In this lies the utmost danger for the humanity. The second limit of modernity is the destruction of humanity itself. 
"Living labor" is the other essential mediation of capital as such; the human subject is the only one that can "create" new value (surplus value, profit)...The third limit of modernity is the possibility of the subsumption of the populations, economies, nations, and cultures that it has been attacking since its origin and has excluded from its horizon and cornered into poverty... I want to emphasize that the globalizing world-system reaches a limit with the exteriority of the alterity of the Other, a locus of "resistance" from whose affirmation the process of the negation of negation of liberation begins ${ }^{20 "}$.

Dentro de esta perspectiva política, se encuentra el esbozo de la concepción política de Hegel. En palabras de John Milbank ${ }^{21}$, la primera acusación que se le puede hacer, al sistema de Hegel es la de que su "dialéctica" no pasa de ser una nueva variante de la política y de la economía política modernas; el pensamiento negativo de Hegel habría tenido menos éxito que el positivismo en lo que respecta a la superación del liberalismo y de las teorías económicas, así como de la heterogeneidad. Para el autor, la lógica hegeliana sería apenas otra "economía política" y, por tanto, inevitablemente, una teodicea más que otra cosa.

Milbank se ocupa, en la primera parte de la obra citada, sobre la teoría política moderna, que se estructuró, según él, en tres grandes negaciones: La primera negación sería la de la "poiesis barroca", esto es, la idea de que el hacer humano no es una cuestión meramente instrumental y arbitraria, sino que está considerado como una posibilidad que se abre hacia lo transcendente. La segunda negación tiene que ver con la doctrina cristiana de la Creación, a favor de un retorno a la mitología antigua de la acción racional como "inhibidora del caos". Y, finalmente, de la ética política aristotélica, con sus nociones centrales de praxis, virtud y prudencia.

El impacto de la globalización de los últimos tiempos ha concernido a la vida del ser humana en todas sus dimensiones. Para que podamos conocer y dar las debidas dimensiones de la globalización en la sociedad contemporánea, necesitamos valorar el desarrollo histórico por el cual pasaron nuestras sociedades y comunidades en los períodos de la historia mundial más reciente. Deberemos hacer un análisis de lo que representa la globalización para la vida socioeconómica, política, y principalmente, en lo que respecta a los valores nuevos que sc instauran de modo significativo en la vida de la persona humana, en cuanto individuo y como sujeto protagonista de su historia y como miembro de la sociedad. El proceso de globalización se desarrolla desde el inicio de la Modernidad como una nueva visión que las ciencias naturales brindan al medio humano, así como las nuevas conquistas que tenemos del mundo geográfico en virtud de la expansión y, conquista europeas iniciadas ya al final de la Edad Media y consolidadas en la Edad Moderna, que posibilitaron al hombre europeo alcanzar nucvas mercancías y, al mismo tiempo, paladear algo diferente como un nuevo conocimiento, así como también nuevos valores que se introducen en la vida de 
la comunidad a partir de la experiencia del totalmente Otro, como ocurrió en el caso de la conquista de nuevos territorios y de la Conquista Espiritual iniciada por la Península Ibérica en la aurora de la Edad Moderna. Podemos encarar la globalización de la experiencia histórica de violencia encontrada en las relaciones económicas de explotación como estructura del nuevo sistema mundial. "The despotic dominance over the bodies of the new colonialism servants was structured on the basis of an economic system which was founded not even an unequal exchange, but on the simple extraction, pillaging, or illegal appropriation of all resources that could be exploited through military dominance. Indians were sent by means of the system of encomiendas -a system characterized by gratuitous labor - to work in the fields and later work in the haciendas (farms) where they received fictious salaries; they were sent to mines where they labored their lives away in the mita. Africans were commodified as slaves, used and slaughtered like animals (treated as pure merchandise deprived of fundamental rights such as marriage, paternity or any other right known to humans). ${ }^{22}{ }^{23}$ En la historia de la Modernidad, se pucde esbozar cómo la globalización indujo una nueva especie de violencia, tal como la dominación política y los límites del poder político impuestos por los Estados modernos y emergentes en la primera fase de descolonización, principalmente ${ }^{24}$, en América Latina.

Por un lado, podríamos hablar del violento impacto económico que la globalización ha causado, de los límites del poder que se han impuesto a los gobicrnos de los Estados de la actualidad, que se caracterizan por las enormes crisis de democracia y de identidad cultural, así como de las nuevas formas de crisis políticas provocadas por el modelo de economía de las empresas transnacionales. Esta realidad actual nos presenta algunos desafíos enormes: la difusión de una nueva escala de valores, la ausencia de utopías, la juventud actual que carga con prolundas desilusiones en relación con la totalidad del sistema mundial, la aparición y el fortalecimiento del capitalismo transnacional, el conflicto entre la globalización y la cultura regional, la cultura de la sumisión y el aparecimiento de la conciencia ccológica más incluyente. Por otro lado, hemos de comprender el desarrollo del capitalismo vinculado a la expansión de la economía de mercado de corte liberal y del espacio de vigencia entre el capital y el trabajo asalariado, como una nucva dimensión de la vida económica y de la fundamentación de la política en términos regionales $\mathrm{e}$ internacionales ${ }^{25}$.

El proceso de globalización entendido actualmente, se forma a partir del concepto de desarrollo económico iniciado con las altas inversiones de capital y de tecnologías realizadas inmediatamente después de la Segunda Gucrra Mundial y que alcanza las metas de modernización de las décadas de los 60 y 70 y cue se extenderá por períodos más largos, irreversiblemente y por mucho ticmpo hasta incluir en su esfera a todos los países y mercados. A cste capítulo decisivo de la historia se suma, en la última década del siglo xx la derrota de la civilización ${ }^{20}$, el lin de la guerra fría con la quichra del socialismo real y la instauración del 
modelo de cconomía de mercado como forma delïnitiva y alternativa exclusiva de historia, con la teoría de que la historia llegó a su fin. "The period after the Second World War was a period of hope for many people. There were those who believed in socialism and thought it was being built in the Soviet Union and Eastern Europe and in part of Asia. There were who believed in democracy and freedom and thought we were on the way to achieve them. There were the nations of Asia, Africa, and Latin America moving more rapidly than before to independence. Today', most of these hopes have collapsed under the assault of a global transnational imperialism." $272 \mathrm{k}$

Este sistema mundial alcanzó los objetivos últimos de la modernización introducida en las décadas de 1960 y 1970 en América Latina y en algunas partes de Álrica y Asia. Los regímenes militares de Latinoamérica se sustentaron por la doctrina de seguridad nacional. Esta política extendió su dominio sobre los países y los mercados. Bajo estas perspectivas, podemos añadir los sucesos del fin de la guerra fría y, además, la quiebra del socialismo real en la Unión Soviética, con su desmantelamiento total y la introducción definitiva de la cconomía de mercado como forma definitiva de vida, como alternativa exclusiva de la hisıria bajo la tcoría del lin de la historia, proceso que desencadenó los conflictos más diversos, centrados en la preponderancia étnica y la identidad cultural. Immanuel Wallerstein observa: "La destrucción del muro de Berlín y la subsecuente disolución de la Unión Soviética se celebraron como muestras de la quicbra del comunismo y del colapso del liberalismo y nuestra entrada definitiva en el mundo del posliberalismo". ${ }^{29}$

Así, en la concepción de la globalización se incluiría el proceso que los mercados realizan superando todas las lronteras políticas, sociales, étnicas y culturales, hasta la universalización del consumo dentro de una unidimensionalidad y de una unidireccionalidad de aldea global.

En el presente contexto de globalización, las crisis económicas del capitalismo se verían siempre como crisis ya vencidas y que serían algo superado y perteneciente al pasado. Así, las políticas anticíclicas del tipo de análisis histórico-económico de Keynes, siempre serían capaces de minimizar los efectos más destructivos de una crisis y de canalizar sus energías negativas para la renovación y el perfeccionamiento de las condiciones técnicas y organizacionales de producción. Esto equivaldría casi a que los países atrasados en su desarrollo científico-técnico y económico pudiesen todavía recuperar parte de la historia del tiempo perdido. Por otra parte, se menciona que el sistema económico y político actual habrían adquirido la capacidad de distribuir las riquezas con mayor justicia social, extendiendo sus beneficios incluso para las clases sociales otrora empobrecidas, como es el caso del proletariado. Los trabajadores, el proletariado mismo, el que carece de capacitación técnica, tendrían todas las formas de participación efectiva en todos sus derechos, en la realización de las condiciones de trabajo y de las condiciones de una vida feliz. El proletariado se presenta ahora como si estuviera integrado de una

Éłica y política: Entre la utopia y la justicia social 
forma más justa dentro de la órbita del capital, no por el hecho de ser un consumidor importante, sino por el hecho de poder negociar sus condiciones de trabajo y salariales y de poder, incluso, participar ef ectivamente de las ganancias de las empresas. Con esta nueva situación histórica de la relación del trabajador con el capilal, implicó que el sindicalismo tuviera un nuevo carácter, con menor fuerza icleológica y de lucha por la defensa del trabajador. Este es un nuevo fenómeno, clesaliante socialmente hablando, que posee dimensiones universales. Es decir: el nuevo sinclicalismo está inserto en el mismo proceso económico de globalización cle la economía y de la ideología. Todos los procesos de unidades políticas se han visto insertos en una concepción global merced a ese nuevo proceso.

Estc modelo económico está concebido, actualmente, con la denominación (le capitalismo tardío, al cual se suma un modelo administrativo y político y es conccbido como tal por la nueva denominación de socialdemocracia, o del llamado modelo económico del neoliberalismo. Y el modelo económico se presenta como una superación del campo del conflicto surgido de diferencias ideológicas, en la distribución de la justicia social y con el poder de superar todas las posibilidades de cualquier crisis cconómica.

Desde este punto de vista — cl de la unidimensionalidad económica y el de la unidireccionalidad ideológica- la globalización se presenta como un gran pcligro, pues muchos problemas sociales provenientes de la economía globalizada sc agravan cada vez más en todos los sectores de la sociedad. La globalización, entendida como una fábrica de perversidad. El hambre aumenta y devasta inmensas regiones del Planeta; el desempleo es incontrolable; los salarios son cada vez. más desvalorizados y humillantes; el sueño de la calidad en la enseñanza está casi por perderse de vista; las enfermedades surgen y se expanden con rapidez; por la geografía de la miseria, el hambre y la mortalidad infantil aún reinan soberanamente en muchas regiones del Planeta, aumentando sus efectos devastadores. Al revés de la idea global y el acortamiento de las distancias, lo que ocurre, entreIanto, es la globalización de una humanidad egoísta, competitiva, consumista, corrupla. La unicidad de la técnica, la convergencia de los momentos y conocimienlos clel plancta, son las bases técnicas de las cuales se sirve la globalización perversa. Sin embargo, esas técnicas pueden utilizarse de otra forma y para otros fines. Así, podemos considerar de manera optimista algunos fenómenos emergentes de esta era globalizada. Comenzando por la enorme mezcla de etnias, culturas, gustos, permitiendo compartir filosofías, en detrimento del eurocentrismo presente en la historia de la filosofía moderna; y, actualmente, comenzamos a aceptar y reconocer los muy distintos centros de filosofía y ya no nos regimos más por el centrismo y el fundamentalismo. De esta manera, surge una cultura popular basada en la diversidad, sirviéndose de los medios técnicos antes exclusivos de la cultura de masas, ejerciendo sobre ésta cierta actitud de venganza. La globalización del modelo económico, como sistema mundial, se concentra en la fuerza y el poder que están siempre inherentes al poder económico y de oferta de una empresa

\section{8}


uransnacional. Esta nueva figura económica: la empresa transnacional, no concentra tan sólo un poder económico, sino un poder político, que manipula el poder delimitado de las democracias, de los representantes legítimos del pueblo y obliga a los mismos políticos en su responsabilidad moral para administrar la justicia social. Se hace énfasis, aquí, en la dificultad moral que acompaña a la política de los países afectados por el lado perverso de la Globalización, principalmente, en la realización de los programas esenciales y fundamentales para la población: crear empleos para lodos, elevar el nivel de instrucción, adecuar un modelo educativo a los problemas de la sociedad contemporánea y de cara a los desafíos del futuro, crear un sistema de salud justo y garantizar el bienestar para cada ciudadano.

En lo tocante a la crítica social del modelo económico de la globalización, sería sumamente necesario discutirlo a partir de las principales teorías críticas del capitalismo y del socialismo del siglo XX, principalmente, lo que la Escuela de Frankfurt hizo a través de sus representantes más sólidos, tales como Horkheimcr, Adorno, Marcuse, Hans Jonas y, recientemente, por Jurgen Habermas, con rclación a la sociedad postcomunista, señalando perspectivas realistas y soluciones a los problemas que heredamos del siglo $\mathrm{xx}$.

En Herbert Marcuse ${ }^{30}$, se esbozaba ya una nueva forma de relación del trabajador para con la empresa, a partir de la nueva consciencia que el trabajador va asumiendo. La gran concepción de esta perspectiva de Marcuse sería la permanencia de un modelo económico que integra a las clases trabajadora y burguesa, y que llegan a ser un modelo de capitalismo en el que la burguesía y la clase obrera estarían envueltas en una forma de capitalismo que mantiene las conquislas industriales, tecnológicas y de bienestar sin acentuar más la dimensión de lucha de clases. Con eso, la relación de los críticos ha sido diferente con la nueva situación de los trabajadores. Como la dimensión de la lucha de clases habría desaparecido, no existiría más la necesidad de una teoría crítica para la situación específica de los trabajadores. Éstos tienen una nueva amenaza más sobre sus cabezas: el desafío tecnológico y el desempleo. Ya no importa mucho el concepto de alienación en relación con el trabajo y el capital, sino el concepto de alienación. Según su análisis ---que se basa más en el concepto de la fenomenología de la acción del Estado para solucionar los conflictos como una experiencia

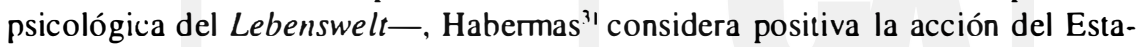
do para solucionar los conflictos que se instauran en las clases trabajadoras. Serían soluciones siempre más reformistas que otra cosa y se trataría de adecuar el mundo del trabajador a las situaciones del Estado.

Así como Habermas, existen muchos otros críticos contemporáneos que creen que el Estado tendrá todas las condiciones para responder por la responsabilidad de la justicia social. "2 Esta tesis es defendida por Jorge Grespan, profesor del Departamento de Historia de la Universidad de São Paulo. 
Teóricamente, verificamos la producción de un nuevo discurso que se vuelve importante por el hecho de revelar una universalidad empírica que deja de cstar tan sólo en elr pensamiento de los filósofos y pasa a ser el resultado de las experiencias de cada ser humano situado en el mundo de la vida.

Como una nueva crítica al modelo de globalización económica, voy a utilizar la crítica realizada por Milton Santos, crítico de la globalización a partir de la perspectivas de los países del Tercer Mundo. La lectura deberá hacerse desde dos dimensiones: por un lado, los impactos terribles que la globalización ha provocado, principalmente, cuando se trata de un modelo meramente económico de cxclusión social, pero, por otro lado, de un modelo que quicre pasar por ser el pensamicnto único en la consciencia universal.

La globalización requiere una interpretación multidisciplinaria del mundo contemporáneo, en la que se realza el papel de la ideología en la producción de la historia y muestra los límites de su discurso frente a la realidad vivida actualmentc por la mayoría de naciones.

Por un lado, encontramos la tiranía de las informaciones y, por el otro, la dominación del dinero, que se presentan como pilares de una situación en la que cl progreso técnico es aprovechado por un pequeño número de actores, participantes globales que actúan en su beneficio exclusivo. Una nueva situación histórica sc ha creado cuyo resultado es la profundización de la competitividad, la producción de nuevos totalitarismos, la confusión de los espíritus y el empobrecimiento galopantc de las masas, por cuanto los Estados se vuelven incapaces de regular la vida colectiva. Es una situación insostenible. En el libro de Milton Santos encontramos un análisis profundo de las transformaciones que ocurren en Asia, África y América Latina, así como en los movimientos populares protagonizados por los sectores más pobres de la población. El autor habla de una crisis estructural ${ }^{33}$. El proceso de la globalización perversa tiene sus bases en la unidad totalitaria de la técnica, de la convergencia del tiempo y del conocimiento del mundo ${ }^{34}$. Delante de cstas circunstancias históricas actuales, podremos ver brotar la simicnte de una cvolución positiva, que debcrá conducir al establecimiento de otra globalización posible, cuando las clases y los grandes grupos de individuos, ahora excluidos, scan los protagonistas y sujetos-autores en la construcción de un nuevo universalismo bucno y justo para todos los pueblos y personas. Esta dimensión utópica deberá alentarse en todas las circunstancias contemporáneas. "Nadie persiguc por gusto una utopía, y menos todavía hoy, cuando todas las energías utópicas parecen haberse agotado definitivamente. La idea de una política que recupcre su primacía sobre los mercados ni siquiera ha llegado a plasmarse como "proyecto", y no existen tampoco dentro de las ciencias sociales esfuerzos teóricos que vayan en esta dirección. Tal proyecto debería al menos proporcionarnos ejemplos de un posible equilibrio de intereses exigible a iodos los participantes, así como el esbozo de procedimientos y prácticas adecuados para ese fin. Es comprensible la resis- 
Icncia de las ciencias sociales ante un proyecto de régimen político Iransnacional que transforme toda política en una política interior mundial. Sobre todo si tenemos en cuenta que un proyecto como éste tendría que justificarse a partir de la actual constelación de intereses de los Estados y sus respectivas poblaciones y ser llevada a cabo por poderes políticos independientes." 3 s

\section{El principio ético: Entre la utopía y la justicia social}

Dchemos alentar a los grupos de derechos humanos como una ćlica de emergencia para que encuentren un lugar en la sociedad como una lucrza revolucionaria en cuanto sujetos prolagonistas de la propia historia de sus derechos individuales y de los derechos de la autodeterminación como pucblo. Esos derechos se entienden tanto como una crítica al sistema mundial de la economía, así como el derecho a ser diferentes. Surge también una conciencia política y económica en relación con la siluación del nuevo orden mundial. Con el desarrollo de la globalización económica y política existe, al mismo tiempo, el surgimiento de una conciencia de la exclusión social y del clamor por una participación real y electiva de todos en las mesas de negociaciones, en las iguales condiciones. Los puchlos que viven aclualmente en una exclusión total, exigen volverse sujetos le su propia historia, como protagonistas de la vida en cuanto sujeto individual y social. Desde esta perspectiva, se lundamenta la ética de la libcración que, según Enrique Dussel, debe justificar y complementar dos principios lundamenlales en la perspectiva ćlica: Los principios de lo material y de lo formal. "Liberation Ethics must still foreward that the function of ethics in relation to globalization does not end in the provision of discursive regulations needed to reach a consensus from which specific measures can be implemented. Its function does not end either in offering abstract guidelines for the principle of reproducing and helping to develop the life of any human subject - a principle that is universal and from which the discursive principle functions as its moral mediation of application. Liberation Ethics nust still take into consideration the factibility of the decisions to which it arrives based upon the fulfillment of the two principles already discussed: the material and the formal principles" $36,17$.

Según José Luis Gómez. Martínez, necesilamos un camino intercultural, que Jeberá tener un carácter interdisciplinario para analizar la globalización. Solamente en la complejidad interdisciplinaria podemos superar la realidad cotidiana por medio del pensamiento. La globalización crea lambićn una nueva conciencia sobre el derecho a las dilicrencias. ${ }^{1 \times}$ La filosolía intercultural es una nueva orientación en el cstudio de la lilosolía y sirve como respuesta para los grandes desalíos ćlicos en la era de la globalización. Toda cultura ha desarrollado caminos lípicos para lilosofar y of́recer explicaciones peculiares del mundo, de la naturaleza humana y de los derechos, así como de las relaciones múltiples entre los seres humanos. En cuanto el período de la globalización, muchos aspectos de la vida humana que surgen en el problema aclual, la filosofía necesita de 
nuevas formas y mélodos para postular un mundo más justo con características interhumanas. La filosofía intecultural habrá de confrontarse con las caracteríslicas del mundo presente: la pluralidad de las culturas, del mundo de la vida y de los intereses tales como la movilidad existencial y social, y de las sociedades politizadas. En la filosofía intercultural, podemos encontrar procedimientos de un "polílogo", para superar el universalismo centralista y el particularismo separatista de la filosofía. ${ }^{31}$ Gracias a la concepción positiva de globalización, es posible contar con una nueva posibilidad de respeto a los derechos humanos y de reconocimiento de la alteridad de las personas y de los pueblos excluidos. La globalización exige un propósito activo y un camino sobre el diálogo intercultural en torno a la naturaleza, a la realidad y sobre el nuevo mundo que se vislumbra en cl horizonte de la historia. No estamos en el fin de la historia. La verdadera historia de la humanidad está en sus albores. Esta historia es la historia de las voces del pucblo y se encuentra en la exclusión social, económica y política. La verdadera historia recuperó la dimensión dialéctica, una nueva dimensión conflictiva del proceso de ascenso de la conciencia social. La historia se vuelve actualmente la historia universal de la conciencia de la participación plena en el pluralismo y en cl multiculturalismo, acrecentando una verdadera dimensión humana. ${ }^{40}$ Esta es también la historia del pobre, del pauper post-festum, según Marx en la interpretación de Enrique Dussel, de este pobre que clama por justicia en una interpelación ética. El reconocimiento político de la alteridad del otro podrá trabajarse por medio de una perspectiva ccumćnica de los elementos que posee la infinita responsabilidad ética. Es como si se tratara de una nueva conjugación del concepto de Utopía y de realización de la justicia social. En el pasado, la comprensión de la historia universal no era más que la visión pretenciosa, ideológica y política, de los países dominantes y de las culturas en relación con las demás culturas y de los pueblos que eran considerados como bárbaros e irrelevantes. ${ }^{41}$ El proceso dc globalización se asoció siempre, durante la expansión europea y de la Revolución Industrial, al sistema capitalista como sistema mundial, pero, actualmente, presenta otros aspectos tales como la polarización creciente, la exclusión social, la globalización del capital, la segmentación del trabajo, la supremacía del capital especulativo como causa de la nueva crisis del capitalismo ${ }^{42}$, aceleración de las redes de comunicación y de sumisión del capitalismo bajo la hegemonía neoliberal.

Nuestro mundo es un mundo complejo, volcado para la creación de nuevas relaciones viables por la red de comunicación. Esta comunicación es un modo cle producción de nucvos clementos para la vida humana y también constituyc una contribución para el reconocimiento de los derechos humanos. Es necesario crear un nucvo paradigma para entender los valores de la dignidad del ser humano y la responsabilidad inlinita para con el otro, de la subjetividad que necesita verse traducida para formar el concepto de una comunidad de comunicación de la vida. ${ }^{43}$

En cl transcurso de la historia contemporánea, podemos ver un movimiento ccuménico, que es la marcha por el reconocimiento del derecho a la propia identi- 
dad del individuo y de los grupos sociales. La postmodernidad se caracteriza por el énfasis de un nuevo espacio del multiculturalismo, como paradigma de la filosofía —el reconocimiento de la dimensión infinita del Otro.

La globalización no es meramente un fenómeno económico, como ya fue dicho anteriornente, sino que se trata de la forma de un nuevo desarrollo de las relaciones humanas para los próximos períodos de la historia. Solamente en la actualidad, la humanidad se puede identificar como una totalidad y reconocer su unidad. El excluido quiere tener voz en un mundo político. Este deseo es objeto de estudio para muchos autores, principalmente de latinoamericanos que están cnfrascados en definir y discutir la temática de la identidad cultural. La democracia será real solamente a través de un sistema efectivo de justicia universal y a través de un reconocimiento universal de los derechos humanos y de la fundamentación de la subjetividad y de la libertad humana en el precepto de justicia. Este concepto es fundamental en el pensamiento de Emmanuel Levinas, en cl cual podemos llegar a una mejor comprensión del nuevo pensamiento sobre la universalidad de la cultura y también de los valores de la dignidad humana. "La conciencia primera de mi inmoralidad no es mi subordinación al hecho, sino al Prójimo, al infinito. La idea de totalidad y la idea de infinito difieren precisamentc por eso: La primera es puramente teorética, la otra es moral" ${ }^{\prime 4}$ El concepto de totalidad e infinito y, asimismo, el método fenomenológico de la filosofía de Emmanuel Levinas son elementos que desafían a la filosofía en la construcción de los elementos para una crítica renovada y constante al sistema de la globalización, así como para responder a los desafíos de la reconceptualización de la política y para crear posibilidades de paradigmas válidos para la articulación de la justicia social.

Como crítica postmoderna de la política, conviene introducir el concepto de la ćtica de la interpelación, que considera la plenitud del derecho de la alteridad del otro absulutamente otro como protagonista liberador de la historia. El mismo concepto scrá innovador para hacer una reflexión amplia sobre el sentido de la política en tiempos de crisis total. Esta crisis que vivenciamos deberá ser comprendida como una crisis de valores que se instauró en la sociedad contemporánca, en la cual se vuelve más difícil delinear una concepción válida de paradigma político y se acentúa con mayor profundidad el carácter de la relación entre política y justicia social.

Esta perspectiva requiere una mayor profundización de la crítica desencadenada por el pensamiento de la Escuela de Frankfurt, cuyo representante es Walter Benjamin, y la nueva perspectiva de la discusión del carácter político para la era de la globalización, presentada por Immanuel Wallerstein. Según la crítica de los autores mencionados, es necesario desarrollar con audacia y criterios una perspectiva académica sobre la dimensión política. Principalmente, en la actualidad, la dimensión de la utopía deberá ser recobrada y sustentada como una forma de 
esbozar una crítica consciente a la situación conflictiva y contradictoria. Se desarrolló, en las últimas décadas, un sentimiento que podríamos llamar antiutópico.

Reina una gran contradicción en el sistema mundial contemporáneo, como lo detine muy bien George Soros, para quien existe actualmente un tremendo desequilibro entre las tomas de decisiones individuales, tal como se expresa en los mercados, y la toma de decisiones colectivas, tal como se expresa en la políti$\mathrm{ca}^{45}$. La sociedad política y la sociedad económica se encuentran muchas veces como si estuviesen huérfanas de utopía. La utopía, entendida como el sueño de las generaciones más antiguas que contemplan en la retrospectiva existencial posibilidades que aún pueden ser restauradas en la línea de la consciencia histórica, así como para alentar en la juventud visiones que trasciendan la mediocridad de las propuestas que los sistemas sociales, principalmente la educación, presenta a los jóvenes. Aquí es más que fundamental y necesaria una utopía en sí, como utopía crítica de la vida y de la condición humana del presente y que nos lancemos en dirección de los mayores desafíos. Esto, concebido en una perspectiva realmente válida como un proyecto calcado de la realidad, en la viabilidad social y no como algo muy romántico e ilusorio, de carácter alienador cn extremo, se deberían concebir proyectos altamente democráticos que contemplen las posibilidades de la identidad $\mathrm{cl}$ individuo como sujeto y como protagonistas de su historia dentro de la identidad cultural. Se comprende aquí la necesidad de la dimensión histórica de la utopía que posibilite esbozar proyectos viables para que el ser humano pueda encontrar plenamente la felicidad y la prudencia en su vida social. Deberán ser propuestas alternativas de políticas que promuevan - además de la justicia social, por el lado de la concreción del materialismo histórico- la recuperación de la dignidad de la vida y del propio imaginario social de cada ciudadano. Y, como perspectiva última, sería interesante consullar a nuestros niños y adolescentes, pasándoles un cuestionario de tan sólo una pregunta: "¿Qué mundo les gustaria heredar a ustedes de nosotros los adultos, para asumirlo y'venciarlo, con plena dignidad humana, en los próximos años?".

\section{Bimliografin:}

Amin. S. El Capitalismo en la Era de la Globalización. Barcelona: Paidos, 1999.

Assmann, Hugo \& Hinkelammert, Franz. A Idolarria do Mercado. Petrópolis: Vozes. 1989

Benjamín, Walter. Obras Escolhidas. Vol. I Sāo Paulo: Brasiliense, 1985

Bhabha. Homi K. The Location of Culture. New York: Routledge, 1998

Bridges. Thomas. The Culure of Citizenship. Inventing Postmodern Civic Culture. New York: State University of New York Press, 1994.

Cohen. Richard A. Eleventions: The Height of the Good in Rosenzweig and Le'inas. Chicago: The University of Chicago Press, 1994.

Critchley. Simon. The Ethics of Deconstruction: Derrida and Levinas. Edinburgh: Edinburgh Universily Press, 1999. 
Dussel, Enrique. Ética da Libertação. Na idade da globalização e da exclusão. Petrópolis: Vozes, 2000.

\section{Las Metáforas teológicas de Marx. Navarra: ed. Verbo Divino, 1993}

. Globalization and the Victims of Exclusion: from a Liberation Ethics

Perspective. In: The Modern Schoolman, LXXV, January, 1998 pp. 119-155.

Fanon, Frantz. The Wretched of the Earth. London 199().

Feuerhach, Ludwig. Princípios da Filosofía do Futuro. Lisboa: Edições 7(), 1988.

Fornet-Betancourt, Raul. Hacia una filosofia intercultural latinoamericana. San José-

Costa Rica : DEI, 1994.

Kulturen der Philosophie. Aachen: Verlag der Augustinus, 1996.

García Canclini, Nestor. Hybrid Cultures: Strategies for Entering and Leaving Modernity. Minneapolis, 1995.

Galcano, Eduardo. Patas Arriba. La Escuela del Mundo al Revés. Montevideo, 1998

Górnez-Martínez, José Luis. La Posmodernidad y el Discurso Antrópico de la Liheración.

In: Raúl Fornet-Betancourt, Kulturen der Philosophie. Aachen: Augustinus Verlag, 1996.

- La Cultura indígena como realidad. In: Sidekum, Antonio, Corredor de Idéias: Integração e Globalização. São Leopoldo; Unisinos,

20()), pp. 221-261.

Hahermas, Jurgen. La Constelación Posnacional. Barcelona, Buenos Aires, México:

Paidós, 20()()

Hohshawn, Eric. A Erc dos Extremos. Sāo Paulo: Companhia das Letras, 1996.

Huntington, Samuel P. The Clash of Civilizations. Remarking of World Order. New York Simon \& Schuster, 1997

Jameson, Frederic \& Miyoshi, Masan (edit.) The Cultures of Globalization. London:

Duke University Press, 1998.

Kimmerle, Heinz. \& Wimmer. Franz. Philosophy and Democracy in Intercultural Perspective. Amsterdam; ed. Rodopi 1997.

l.evinas. Eınmanuel. Totalidade e Infinito. Lishoa: Ediçōes 7(), 1988.

Llewelyn, John. Emmanuel Levinas. The Genealogy of Ethics. London and New York: Roulledge, 1995.

Marcuse. Herbert. A Ideologia da Sociedade Industrial. Rio de Janeiro: Zahar Editores, 1969.

Marx. Karl. O Capital. Rio de Janciro : Civilizaçāo Brasileira, 1968

Milhank, John. Teologia e Teoria Social. Sāo Paulo: Loyola, 1995

Missac. Pierre. Passagem de Walter Benjamin. Curitiba: Iluminuras. 20)1

Oliveira, Manliredo Araújo de. Ética e Sociabilidade. São Paulo: Loyola, 1993.

Peperzak. Adriaan. To the Other. West Lafayelte, Indiana 1992.

. Ethicis as First philosophly: New York: Routledge. 1995.

Crilchley, Simon \& Bernasconi, Rohert. Emmamuel

Lerinas: basic philosophical Writings. Indiana: Indiana University Press, 1996.

Pinto. Álvaro Vicira. Ciência e lixistência. Rio de Janciro: Paz c Terra, 1979.

Rohertson, Roland. Glolalization: social Theory and Global Culture. London 1992.

Sibato, Ernesto. La Resistencia. Buenos Aires: Seix Barral, 200)(

Santos, Milton. Por uma outra globalizaşão: do pensamento ínico ci consciência univerersal. Rio de Janciro/São Paulo: Record. 20()().

Sciannone, J.C. \& Santuc. V. Lo Politico en América Latina. Buenos Aires: Bonum, 1999.

Ético y politica: Entre lo utopio y lo justicio social 
Sen, Amartya. Development as Freedom. New York: Alfred A Knopf, 1999

Sidekum, Antonio. Ética do Discurso e Filosofia da Libertação. São Leopoldo: Unisinos, 1994.

. Corredor de idéias: Integração e Globalizaçāo. São Leopoldo: Unisinos, 2000.

Smith, Adam. Riqueza das naçōes. Curitiba: Hemus, 2001

Soros, George. La Crisis del Capitalismo Global. Buenos Aires: Editorial Sudamerica. 1999.

Taylor, Charles. The Ethics of Authenticity. Cambridge: CBC, 1999

Todorov, Tzvetan. The conquest of America: The Question of the Other. New York:

Harper and Row, 1984.

Wallerstein, Immanuel. After Liberalism. New York: New Press, 1995.

- The End. Minneapolis: University of Minnesota Press. 1999.

Utopistics. New York: The New York Press.

After Liberalism. New York: New York Press, 1995

Wyschrogrod, E. Emmanuel Levinas. The Problem of ethical Metaphysics. The Hague, 1974.

Zea, Leopoldo. Discurso desde la Marginación y Barbarie. Barcelona: Anthropos 1988.

\section{Notas}

I. Walter Benjamin (15.7.1892-1940). Quiero indicar aquí una importante obra sobre el filósofo alemán, pues su autor es Pierre Misac: Passagem de Walter Benjamin. São Paulo, Editorial Iluminuras, 1998.

2. Immanuel Wallerstein (1930). Dirige el Fernand Braudel Center for the Study of Economics, Historical System and Civilization en la Universidad de Binghamton. También tiene una cátedra en la École des Hautes Études en Sciencies Sociales, en París. Entre sus libros principales se destacan: The Modern World System, The capitalist World Economy y Historical Capitalism.

3. Walter Benjamin, Obras Escolhidas. Vol. 1. São Paulo, Brasiliense, 1985, pág. 222.

4. Adam Smith, A Riqueza das Naçōes. Curitiba: Hemus, 200I. Libro IV, cap. 2, p. 203. Marx extrae las consecuencias de Adam Smith. De esta manera, podemos leer en El capital: "La mercadería es misteriosa simplemente por encubrir las características sociales del trabajo propio de los hombres, presentándolas como características materiales y propiedades sociales inherentes a los productos del trabajo; por ocultar, en consecuencia, la relación social entre los trabajos individuales de los productores individuales y el trabajo total, al reflejarla como una relación social existente, al margen de ellos, entre los productos de su propio trabajo. A través de este encubrimiento, los productos del trabajo se convierten en mercancías, cosas sociales, con propiedades perceptibles e imperceptibles a los sentidos". Cfr. Karl Marx, $O$ capital. Rio de Janeiro: Civilização Brasileira, 1968, p. 81

5. Hugo Assmann y Franz Hinkelammert, $A$ idolatria do Mercado. Petrópolis, Vozes, 1989. P. 12.

6. Álvaro Vieira Pinto nos presenta un bello capítulo que trata de la Teoría de la Cultura, considerando la ciencia como un aspecto particular de la realidad general de la cultura. La cultura, tratada como creación humana resultante de la resolución de la contradicción principal del hombre, aquella existente entre él y la naturaleza. Cultura y hominización. Las nociones de "instrumento" y de "técnica". La cultura como producto del proceso productivo. La cultura de producción de los bienes de consumo 
y de bienes de producción. El hombre como bien de producción. El problema histórico de la evolución de la cultura. Acumulación y apropiación de la cultura. La escisión de la cultura en las sociedades divididas en grupos antagónicos. La división social del trabajo. La cultura como mediación de toda realización humana. El autor trata del carácter humano de la existencia del hombre en razón de tener que ser el creador de la cultura, el animal culto. El análisis entre la mediación recíproca entre cosas e ideas. Y la idea como creación humana. Cfr. Álvaro Vieira Pinto, Ciência e Exislência. Rio de Janeiro, Paz e Terra, 1979. p. 119.

7. Karl Marx. O capiral. Rio de Janeiro: Civilizaçāo Brasileira. 1968. P. 45.

8. "Los filósofos se han limitado a interpretar el mundo de diferentes maneras, pero de lo que se trata es de transformarlo". Karl Marx, Tesis sobre Feuerbach, en K. Marx y F. Engels, La ideologia alemana. Montevideo, EPU, 1959, p. 119.

9. Ludwig Feuerbach, Principios da filosofia do fururo. Lisboa, Edições 70, 1988.

1(). Enrique Dussel se refiere a este punto de la siguiente manera: "Todo «acto-de-trabajo» (como todo argumenta el "acto de habla» seriamente comunicativo) ya siempre presupone la «comunidad ideal de productores». Nunca se trabaja sólo para sí, como no puede argumentarse sólo para sí. El Otro es la contrapartida esencial de todo "acto-de-trabajo» ya que el acto-de-trabajo no es sólo un acto técnico (persona-naturaleza), sino práctico (persona-naturaleza-persona); se encuentra entonces subsumido en un «acto de justicia» (y «Justica ad alterum est (la justicia tiende al otro), decían los latinos. La racionalidad económica, desde la praxis productiva (analógicamente, la comunicativa), presupone ya siempre una «comunidad ideal» ilimitada. definida ahora desde la corporalidad necesitante ( «iTengo hambre'»), como Marx lo describió adecuadamente desde los Manuscriros del 44". Enrique Dussel, Las meráforas teológicas de Marx. Navarra, Editorial Verbo Divino, 1993, p. 305.

1 1. "Por lo tanto, también para Aristóteles, el ciudadano es el sujeto de la Polis, como en cl pensamiento moderno, pero es imposible pensar la praxis de ese ciudadano sin hacer referencia a las instituciones "éticas», pues estas son la vida y el ser de los individuos libres: son su realidad". Manfredo Araújo de Oliveira. Érica e Sociabilidade. São Paulo: Edições Loyola, 1993, p. 61.

12. Immanuel Wallerstein, After Liberalism. New York, The New Press, 1995.

13. Ídem, pág. 252: The Agonies of Liberalism: What Hope Progress.

14. Ver, en ese sentido, la obra de Eric Hobsbawn, Era dos exiremos. São Paulo, Companhia das Letras, 1996, que caracteriza al siglo XX como extremo breve y extremo. Su historia y sus posibilidades se edifican sobre catástrofes, incertidumbres y crisis, comparado con el siglo XIX.

15. Walter Benjamin nos presenta en sus escritos esta marca dolorosa y trágica del progreso en una de sus tesis sobre el concepto de historia: "Hay un cuadro de Klee que se llama Angelus Novus. Representa un ángel que parece querer apartarse de algo que mira fijamente. Sus ojos están desorbitados, su boca dilatada, sus alas abiertas. El ángel de la historia debe tener ese aspecto. Su rostro está dirigido hacia el pasado. Donde nosotros vemos una cadena de acontecimientos, él ve una catástrofe única, que acumula incansablemente ruina sobre ruina y las dispersa a nuestros pies. A él le gustaría detenerse para levantar a los muertos y unir los fragmentos. Pero una tempestad sopla del paraíso y se prende de sus alas con tanta fuerza que él no puede volverlas a cerrar. Esa tempestad lo impele irresistiblemente hacia el futuro, al cual él le da las espaldas, por cuanto el montón de ruinas crece hasta el cielo. Esa tempestad 
es lo que llamamos progresom. Op. Cit., p. 226.

16. Ver Karl Marx, $O p$. Cit., p. 81 . Este fetichismo del mundo de las mercancías, según el autor, oculta el carácter social propio del trabajo que las produce.

17. Hugo Assmahn y Franz J. Hinkelammert, $A$ idolatria do mercado. Petrópolis, Vozes, 1989.

18. Según Levinas, actualmente cargamos cada vez más con una consciencia de lo inmoral, por el hecho de que hacemos poco caso de la ética. Ver, de Emmanuel Levinas, Totalidad e Infinito, Lisboa, Ediçōes 70, 1988.

19. Enrique Dussel: Beyond Euroncenstrism: The World-System and the Limits of Modernity. In Frederic Jameson and Masao Miyoshi, The Cultures of globalization. London, 1998, p. 20.

20. «El primer límite absoluto: la muerte de la vida en su totalidad, mediante el uso indiscriminado de tecnología antiecológica, constituida progresivamente bajo el único criterio del manejo cuantitativo del sistema mundial en la modernidad: el incremento de las ganancias. El segundo límite de la modernidad es la destrucción de la humanidad misma. En esto yace el peligro más grave sobre la humanidad. El "trabajo viviente" es la otra mediación esencial del capital como tal; el sujeto humano es el único que puede "crear" nuevos valores (valores de excedente, ganancias)... El tercer límite de la modernidad es la posibilidad de subsumir a las poblaciones, economías, naciones y culturas a las cuales ha venido atacando desde su origen y ha excluido de su horizonte y ha arrinconado hacia la pobreza... Quiero hacer énfasis que el sistema del mundo globalizado alcanza un límite con la exterioridad de la alteridad del Otro, un locus de "resistencia" desde el cual comienza la afirmación del proceso de negación de la negación de la liberación” (N. del T.).

21. John Milbank, Teologia e Teoria Social. São Paulo, Ediçōes Loyola, 1995. P. 194.

22. Enrique Dussel, Globalization and the Victims of Exclusion: From a Liberation Ethics perspective, en The Modern Schoolman, LXXV, January, 1998, p. 131. En esta perspectiva es fundamental lo que Jean-Paul Sartre escribió en el prólogo a la obra de Frantz Fanon. The Wretched of the Earth, London, 1990.

23. «El dominio despótico sobre los cuerpos de los nuevos sirvientes del colonialismo estaba estructurado sobre la base de un sistema económico, fundado, no solamente sobre el intercambio desigual, sino que también en la simple extracción, pillaje o apropiación ilegal de todos los recursos que podrían explotarse por medio del predominio militar. Los indios se enviaban por medio del sistema de encomiendas — un sistema caracterizado por el trabajo gratuito- a trabajar en los campos y luego, a las haciendas, donde recibían salarios ficticios; se les enviaba a las minas donde trabajahan lejos de la mita. A los africanos se les trataba como esclavos, se les utilizaba y se les asesinaba como animales (esto es, eran tratados como simples mercancías, privadas de los derechos fundamentales tales como matrimonio, paternidad o cualquier otro derecho reconocido para los seres humanos)». (N. del T.)

24. Bajo esta perspectiva, resulta innovadora la obra completa de Frantz Fanon y, actualmente, la de Homi K. Bhabha, The Location of Culture. New York, Routledge, 1998.

25. Ver Samuel Huntington, The Clash of Civilizations: Remarking of World Order. New York. Simon \& Schuster, 1997.

26. İdem. p. 245 . 
27. Sherif Hetata, Dollarization, Fragmentation and God, en Frederic Jameson and Masao Miyoshi, The Cultures of Globalization, London, 1998, p. 281.

28. «El período iniciado después de la Segunda Guerra Mundial era un período de esperanza para mucha gente. Había quienes creían en el socialismo y pensaban que se estaba construyendo en la Unión Soviética y en Europa Oriental, así como en parte de Asia. Había quienes creíamos en la democracia y la libertad y pensábamos que estábamos en vía de alcanzarlas. Estaban las naciones de Asia, África y Latinoamérica, que se movían con mayor rapidez que antes hacia su independencia. Ahora, la mayoría de estas esperanzas han colapsados bajo el asalto de un imperialismo global transnacional». ( $N$. del $\mathrm{T}$.)

29. Immanuel Wallerstein, After Liberalism. New York: The New York Press, 1995, p. I.

30. Herbert Marcuse, A ideologia do Sociedade Industrial. Rio de Janeiro, Zahar Editores, 1969.

31. "La lista de problemas ante los que se topa cualquier lector de periódicos sólo puede convertirse en una agenda política si encuentra un destinatario en el que se pueda confiar y que todavía confíe en una transformación de la sociedad como medio para realizar determinados fines. El diagnóstico de los conflictos sociales sólo se transforma en una lista de desafíos políticos cuando las instituciones igualitarias del derecho natural se relacionan con una premisa adicional, a saber, con el supuesto de que los ciudadanos reunidos en una comunidad democrática pueden conformar su medio social y desarrollar la capacidad de acción necesaria para esa intervención. El concepto jurídico de autolegislación debe adquirir una dimensión política hasta transformarse en el concepto de una sociedad democrática que actúa sobre si misma. Sólo entonces se podrá lograr, a partir de las actuales constituciones, el proyecto reformista de realización de una sociedad "justa" o " bien ordenada". Jurgen Habermas, La constelación posnacional. Barcelona, Buenos Aires, México: Paidós, 2000, p. 82

32. "En Europa hasta el siglo XVII se formaron Estados que se caracterizaban por un dominio soberano sobre un territorio; estos Estados tenían una mayor capacidad de recaudar impuestos que las viejas formaciones políticas como los antiguos imperios o las Ciudades Estado. En su función específica de Estado Administrador, el Estado moderno se diferenció del tráfico económico mercantil institucionalizado jurídicamente; sin embargo, en cuanto Estado fiscal, seguía dependiendo de la economía capitalista. En el curso del siglo XIX, el Estado, como Estado nacional, se abrió a formas democráticas de legitimación. En algunas privilegiadas regiones y bajo las favorables circunstancias de la posguerra, el Estado nacional, que entretanto se había convertido en modelo universal, pudo evolucionar mediante una regulación de la economía que a pesar de todo deja intacto su mecanismo interno de autorregulación, hasta convertirse en Estado social. Esta afortunada combinación está amenazada en la medida en que una economía globalizada escapa a la intervención del Estado regulador. Las funciones del Estado social han alcanzado actualmente tal dimensión que sólo pueden ser cumplidas si son transferidas desde el Estado nacional a unidades políticas que en cierta forma alcancen y se pongan al mismo nivel que la economía transnacional". Habermas, Op. cit., p. 74

33. "En este período histórico, la crisis es estructural. Por eso, cuando se buscan soluciones que no son estructurales, el resultado es la generación de mayor crisis". Milton Santos, Por uina outra globalização. Rio de Janeiro, 2000, p. 35.

34. Ver también Frantz Fanon, Op. cit., p. 252. Frantz Fanon creó una nueva metodología para el análisis de la historia: la historia estudiada a partir del punto de vista de 
las víclimas. La misma temática sobre el problema del reconocimiento del otro en cuanto otro es usada por Tzvetan Todorov.

35. Jurgen Habermas, Op. cit., p. 76.

36. Enrique Dussed. Op. cit., p. 151.

37. "La élica de la liberación debe aún dejar en claro que la lunción de la ética en lo locante a la globalización no concluye en la provisión de regulaciones discursivas necesarias para alcanzar un consenso desde el cual se podrían implementar medidas cspecílicas. Su función no termina tampoco en of recer guías abstractas para el principio de reproducir y ayudar a desarrollar la vida de cualquier sujeto humano —un principio que es, por lo demás. universal, y desde el cual el principio discursivo lunciona en virtud de su mediación moral de aplicación. La ética de la liberación llcbe aún tomar en consideración la lactibilidad de las decisiones que toma en base de la satislacción de los dos principios ya discutidos: los principios material y lormal" (N. del T.)

38. Josć Luis Gómez Marlínez, La cultura indigena como realidad intercultural, en AnIonio Sidckum: Corredor de idéias: integrą̧āo e globalizaçāo. S. Leopoldo: Unisinos, 2()(). Ver también La posmodernidad y el discurso antrópico de la liberación, en Raúl Fornet-Betancourt, Kulturen der Philosophie, Aachen, Augustinus Verlag, 1996, pp. $181-194$.

39. Ver, de Raúl Fornet-Betancourt, Hacia una filosofía intercultural latinoamericana, San José, Costa Rica, Ediciones DEI, 1994.

4(). Immanuel Wallerstein dice, con respecto a los derechos humanos: "Necesitamos, en suina, Iomar el concepto de los derechos humanos y trabajar muy duro para aplicarlos de igual manera, tanto para "nosotros" como para "ellos", para los ciudadanos y los cxtranjeros. El derecho que tienen las comunidades de proteger su herencia cultural nunca es cl derecho de proteger sus privilegios". Immanuel Wallerstein, Op. cit., 270.

41. Ver. de Leopoldo Zea, Discurso sobre la marginación y barbarie. Barcelona: Anthropos, 1988.

42. Ver el desarrollo de esta idea en George Soros, La crisis del capiralismo global, Buenos Aires, Editorial Sudamericana, 1999.

43. Ver la discusión acerca de la laclibildad de la ética del discurso en Apel, Habermas y Dussel, en: Antônio Sidelum, Érica do discurso e filosofía da libertação. São Leopoldo, Unisinos, 1994.

44. Emmanuel Levinas, Totalidade e Infinito, p. 70.

45. George Soros, La crisis del capiralismo global. Buenos Aires, Sudamericana, 2000. 